\title{
Histone Deacetylase in Carcinogenesis and Its Inhibitors as Anti-cancer Agents
}

\author{
Dong Hoon Kim, Minjung Kim and Ho Jeong Kwon* \\ Department of Bioscience and Biotechnology, Institute of Bioscience, Sejong University, Seoul 143-747, Korea
}

Received 2 November 2002

The acetylation state of histone is reversibly regulated by histone acetyltransferase (HAT) and deacetylase (HDAC). An imbalance of this reaction leads to an aberrant behavior of the cells in morphology, cell cycle, differentiation, and carcinogenesis. Recently, these key enzymes in the gene expression were cloned. They revealed a broad use of this modification, not only in histone, but also other proteins that involved transcription, nuclear transport, and cytoskeleton. These results suggest that HAT/HDAC takes charge of multiple-functions in the cell, not just the gene expression. HDAC is especially known to play an important role in carcinogenesis. The enzyme has been considered a target molecule for cancer therapy. The inhibition of HDAC activity by a specific inhibitor induces growth arrest, differentiation, and apoptosis of transformed or several cancer cells. Some of these inhibitors are in a clinical trial at phase I or phase II. The discovery and development of specific HDAC inhibitors are helpful for cancer therapy, and decipher the molecular mode of action for HDAC.

Keywords: Anti-cancer agents, Carcinogenesis, Gene expression, Histone deacetylase, Histone deacetylase inhibitors

\section{Introduction}

DNA in eukaryotic cells exists in the form of nucleosome. Nucleosome bears an average of 150 base pairs of DNA that are wrapped around the nucleosomal core. The core consists of the histone octamer, $\mathrm{H} 2 \mathrm{~A}, \mathrm{H} 2 \mathrm{~B}, \mathrm{H} 3$, and $\mathrm{H} 4$, in the dimeric form (Kornberg et al, 1974; Kornberg et al, 1999, Luger et al, 1997). Histones bind to negative-charged DNA through the

\footnotetext{
*To whom correspondence should be addressed.

Tel: 82-2-3408-3640; Fax: 82-2-3408-3334

E-mail: kwonhj@ sejong.ac.kr
}

positive charge of lysine in the N-terminal tail of histones. The interaction between histones and DNA is mainly regulated by the modification of lysine. Among several modifications of histone, acetylation has been highlighted as one of the crucial events that are related to the transcriptional response of the cell. The acetylation of histone by HAT neutralizes the positive charge of lysine, which results in a decreased interaction between histone and DNA. This conformational change in chromatin activates the transcription by promoting the access of transcriptional machineries, such as RNA polymerase, transcription factor, and regulatory complexes to DNA. Reversibly, these events are restored when the histones are deacetylated by HDAC (Davie, 1998) (Fig. 1).

Recent extensive studies on these two enzymes revealed that HDAC plays a key role in carcinogenesis, and the enzyme has been considered as one of the target molecules for cancer therapy. In addition to the transcriptional regulation in carcinogenesis, HDAC plays an important role in several interactions, such as protein-DNA interaction, protein-protein interaction, and protein stability by changing the acetylation state of proteins, including p53, transcription factor, and tubulin. From this point of view, the HDAC role in carcinogenesis and other cellular functions, as well as the current progress on the development of HDAC specific inhibitors, will be reviewed.

\section{HAT/HDAC as Key Regulators in the Gene Expression of Eukaryotes}

Reversible acetylation of histones is catalyzed by two key enzymes-histone acetyltransferase (HAT) and histone deacetylase (HDAC) (Fig. 1). Currently, there are four groups of proteins with intrinsic HAT activity. They are as follows: GCN5 and P/CAF, which are related to the yeast HAT; the cyclic adenosine monophosphate response element-binding protein (CBP) and p300, which act as co-activators for a number of transcription factor complexes; TAF250, a part of the basic transcription complex TFIID that binds with the 
(A)

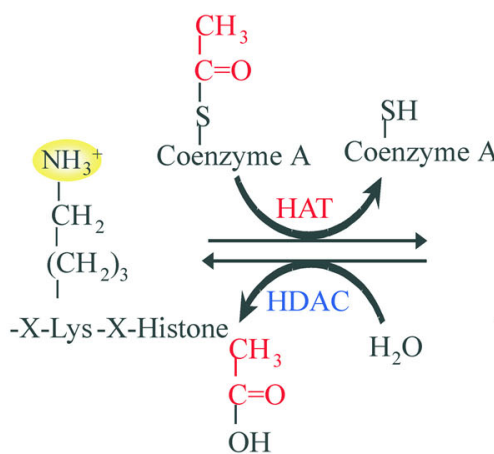

(B)
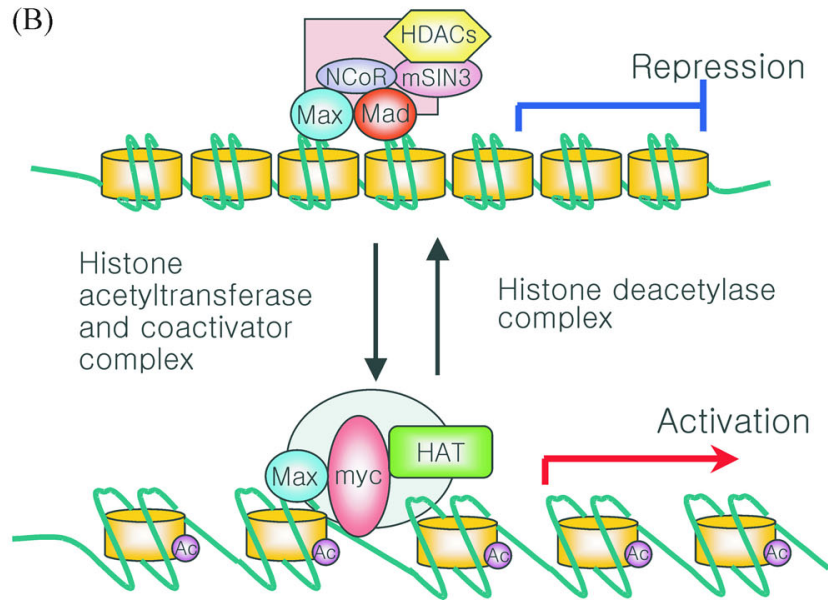

Fig. 1. Role of HAT and HDAC in transcriptional regulation. (A) Histone modification by HAT and HDAC. (B) Regulation of gene-expression switch by co-activator or co-repressor complex.

TATA box; and finally SRC-1 and ACTR, which are coactivators for the ligand-activated nuclear receptors (Bannister et al., 1996; Ogryzko et al., 1996; Mizzen et al., 1996; Spencer et al., 1997; Kouzarides, 1999). Basically, all of these enzymes have covalent modification activity on lysine residue of histone. Also, eleven HDACs have been cloned from humans, and classified into two groups, based on their size and number of catalytic core domains. Class I is the yeast RPD3 homologous, HDAC1, 2, 3, and 8. Class II is the yeast HDA1 homologues, HDAC4, 5, 6, 7, 9, 10, and 11 (Marks et al., 2001) (Fig. 2). Interestingly, Sir2 is identified as HDAC in yeast, and conserved in organisms that range from bacteria to humans. Unlike other HDACs, this enzyme requires nicotinamide-adenosine dinucleoside (NAD) as a co-factor (Lander et al., 2000). A variety of isotypes, and an abundant amount of proteins in these enzymes imply, that these proteins take charge of pivotal roles in cellular functions (Hwang et al., 2000). Because of its role in carcinogenesis, HDAC has been especially interesting. It has become a new and promising target of cancer therapy.
Class 1 (yeast Rpd3p)

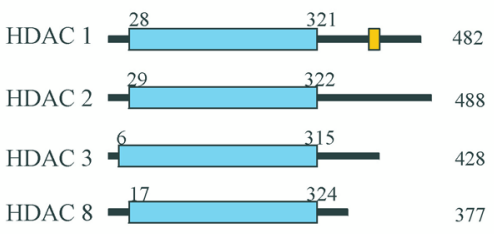

Class 2 (yeast Hda1p)

$\underline{\text { Subclass I }}$
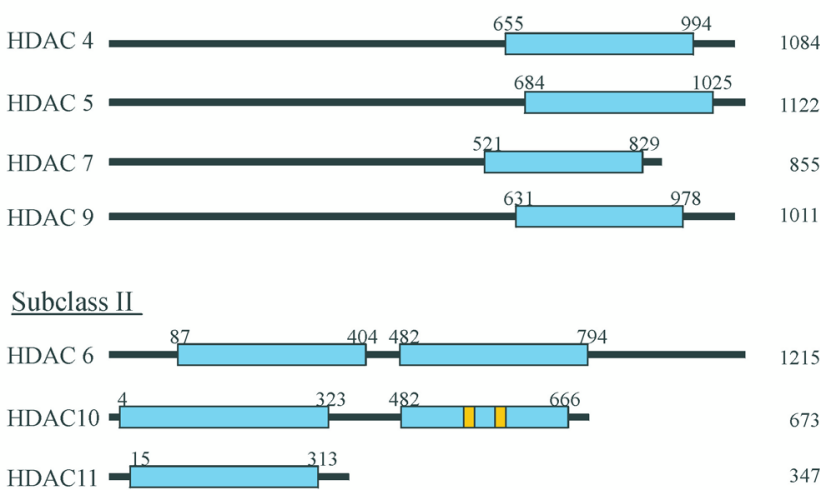

Fig. 2. Classification of HDAC isotypes. Eleven HDACs were identified in mammalian cells and classified into two groups, based on their size and number of catalytic core domain. Class I is the yeast RPD3 homologous, HDAC1, 2, 3, and 8. Class II is the yeast HDA1 homologues, HDAC 4, 5, 6, 7, 9, 10, and 11 . Interestingly, Sir2 is identified as HDAC in yeast and conserved in organisms, ranging from bacteria to humans. Unlike other HDACs, this enzyme requires the nicotinamide-adenosine dinucleoside (NAD) as a co-factor.

\section{HDAC Complexes}

One of interesting features of HDAC is that it requires several other components of multiprotein complexes to perform its cellular activities. In fact, HDAC has usually been found in large protein complexes. Two major HDACs that contain complexes are $\mathrm{mSin} 3 \mathrm{~A}$ and the nucleosome-remodeling histone deacetylase (NuRD) complex. HDAC1 and HDAC2 are associated with the two complexes in most of the mammalian cells. On the other hand, HDAC3 and the class II HDACs are not found in the mSin3A and NuRD complexes (Grozinger et al., 1999). The mSin $3 \mathrm{~A}$ complex controls transcriptional repression by recruiting unliganded nuclear receptor proteins, like NcoR / SMRT or the Mad family of the basic helix-loophelix-zipper proteins (Alland et al., 1997; Heizel et al., 1997; Laherty et al., 1997; Nagy et al., 1997). Other components of the mSin3A complex, except HDAC1 and HDAC2, are mSin3A, SAP18, Sap30, RbAp46, and RbAp48. The functions of mSin3A may act as a scaffold for the complex. SAP30 may 
(A) $\underline{\text { RT -PCR analysis }}$

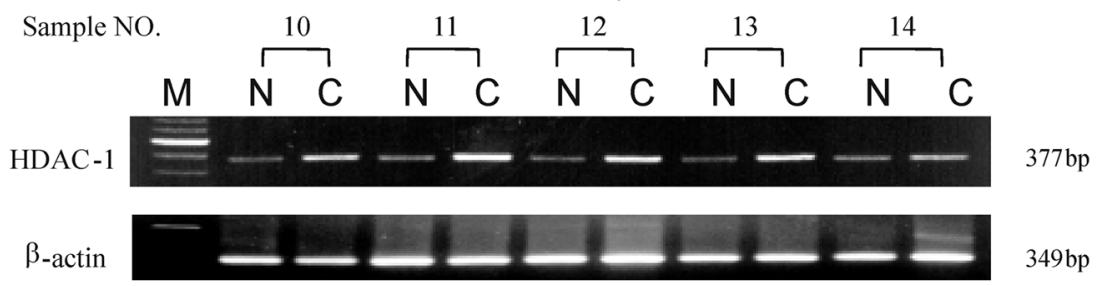

(B) Western analysis

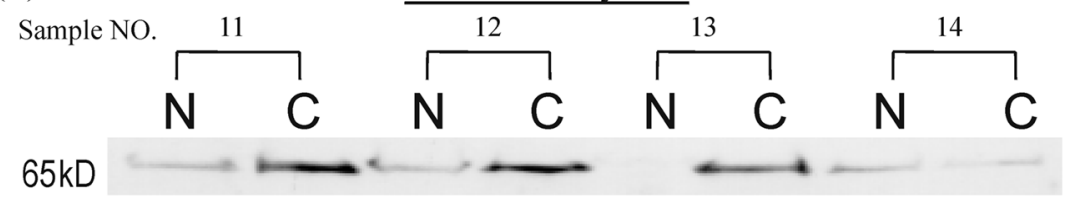

(C)

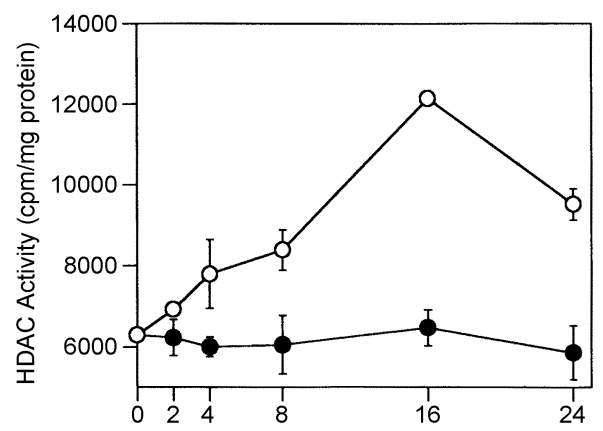

(D)

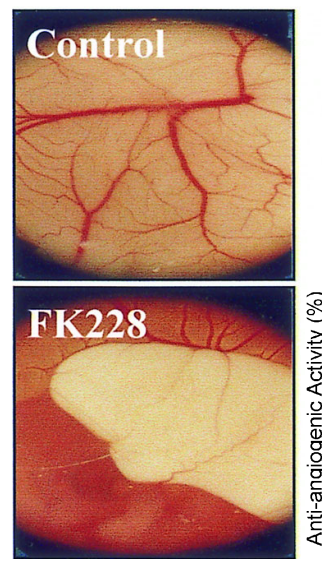

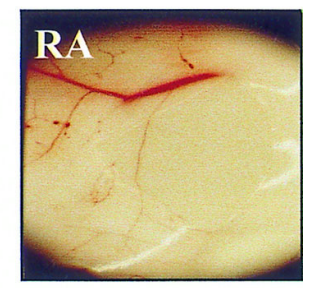

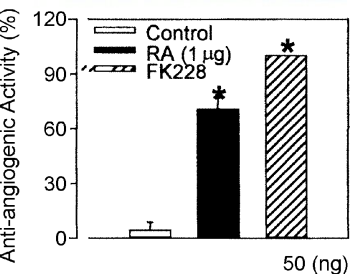

Fig. 3. The HDAC expression in tumor and hypoxia-induced condition (A) HDAC expression in normal and gastric cancer tissues analyzed by RT-PCR. M, marker; N, normal cell; C, cancer cell. (B) Western blot analysis. (C) Time-dependent increase of HDAC activity under hypoxic $(\bigcirc)$ compared to normoxic HepG2 cells. (D) HDAC role in angiogenesis. RA, retinoic acid.

be the target of the mSin3A complex to NcoR-associated repressors. RbAp46 and RbAp48 are histone-binding proteins (Ahringer, 2000). The NuRD complex shares four components with the mSin3A complex (HDAC1, HDAC2, RbAp46, RbAp48) and additionally contains MTA1/MTA2, MBD3, and CHD3/CHD4. The NuRD complex is co-immunoprecipitated with HDAC1 by using the anti-HDAC2 peptide (Tong et al., 1998). Tong et al. demonstrated that the NuRD complex mediates the ATP-dependent nucleasome remodeling and histone deacetylation in transcriptional regulation. Interestingly, the CHD3 and CHD4 proteins in the NuRD complex bear helicase/ATPase domains that are found in SWI/SNF chromatin remodeling factors (Tong et al., 1998). In addition, MTA1 is overexpressed in metastatic carcinoma cells (Toh et al., 1998). It is involved in the migration, invasion, and anchorageindependent survival of immortalized human keratinocytes (Mahoney et al., 2002). These results suggest a crucial role of MTA1 in the HDAC complex for the HDAC function in carcinogenesis. Meanwhile, MBD3 is similar to MBD2, binding to methylated DNA with demethylase activity. MBD2 also binds to methylated DNA, suggesting that the NuRD complex might be involved in DNA methylation (Zhang et al., 1999). Further identification and characterization of the new HDAC complex and its component will help to uncover the HDAC function in the cell.

\section{HDAC Responsive Promoters and Interacting Factors}

Although the eukaryotic chromosomal DNA is packed with histone, HDAC does not appear to universally control the transcription of all genes. The targeting of HDAC to the specific promoters might be mediated by either the direct interaction between HDAC and the HDAC recruiting factors, or by the interaction with other components of the HDACcontaining complex (Fig. 6). For instance, the transcriptional repression of Mad-Max requires the interaction of Mad with mSin3A (Laherty et al., 1997) and MTA1, interacting with HDAC1 in the NuRD complex blocks the ability of oestradiol 

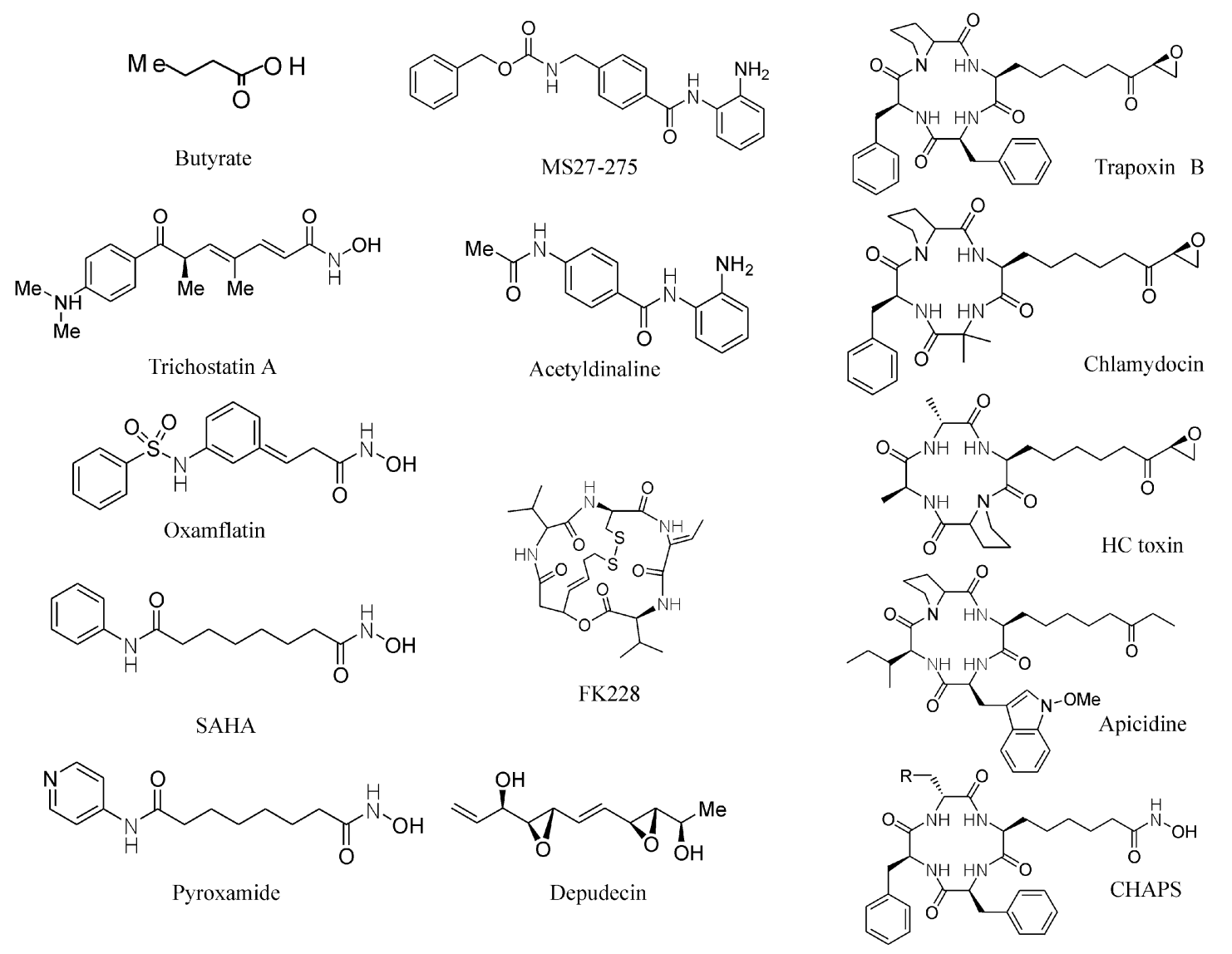

Fig. 4. Chemical structure of HDAC inhibitors.

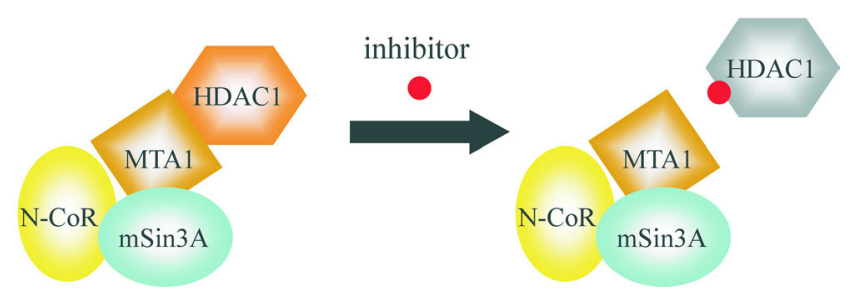

Fig. 5. Schematic HDAC model and co-repressor interaction in HDAC complex and interruption of protein interaction by HDAC complex inhibitor. Recent reports demonstrate that the complex formation between HDAC and its co-repressors is essential for the maintenance of their transcription-regulating function. The disruption of the interaction between HDAC and its co-repressors was induced by the mutation of specific amino acids that are involved in the complex formation. Therefore, the discovery of a compound that will regulate the HDAC complex will be helpful for development of a novel HDAC inhibitor.

to stimulate the ER-mediated transcription (Mazumdar et al., 2001). HDAC complexes are recruited to specific chromosome sites to perform the repression of transcription. For instance, Yeast Rpd3, homologous to human HDAC1, and mammalian HDAC complexes interact with the DNA-binding transcription repressors, YY1 (Yang et al., 1996), Ume6 (Kadosh et al., 1997), and Mad (Laherty et al., 1997). The
$\mathrm{Rb}-\mathrm{E} 2 \mathrm{~F}$ complex that regulates the G1/S transition in the cell cycle is also known to associate with the HDAC activity (Luo et al., 1998). Notably, the methyl-CpG-binding protein 2 (MeCP2) recruits the mSin3A complex, which couples the DNA methylation and histone deacetylation in gene silencing (Nan et al., 1998).

In the case of class II HDACs, HDAC4 and HDAC5 are immunoprecipitated with the 14-3-3 protein, which indicates the shuttling ability of HDAC4/5 between the nucleus and the cytoplasm, depending on the phosphorylation (Grozinger et al., 2000). HDAC4 associates with both NcoR and SMRT in a complex that does not contain $\mathrm{mSin} 3 \mathrm{~A}$ and HDAC1. In addition, HDAC5 is able to interact with MEF2A that controls myogenesis and represses its transcription activator activity (Lemercier et al., 2000). Further studies on the HDACinteracting factors will decipher the molecular mechanism, which underlies the specificity of HDAC in the gene repression and phenotype determination of cells. In addition, the regulation of the HDAC complex formation might be a novel way to control the HDAC function in cells without affecting the enzymatic activity of HDAC (Fig. 5). Furthermore, this approach will provide the specific regulation of the HDAC function in a specific HDAC complex (Kwon et al., 2002). 


\section{HDAC in Carcinogenesis and Angiogenesis}

Extensive studies have shown that the change of the acetylation state of histone is correlated with dramatic biological consequences in the cell. An inappropriate acetylation state of histones causes abnormal outgrowth and the altered pattern of cell death, which leads to neoplasmic transformation (Marks et al., 1978). For instance, the functional mutation in intrinsic HAT has been reported as a major cause of the Rubinstein Taybi syndrome that shows a high malignant frequency (Petrij et al., 1995). Furthermore, HDACs are over-expressed in several tumor cells and tissues (Dhordain et al., 1998; Choi et al., 2001; Pandolfi et al., 2001) (Fig 3, A and B). Notably, a few portions of the total genes are changed by abnormal HAT and HDAC, as described previously. In the neoplasmic state of the cell, the expression of the tumor suppressors, such as p53, p21, and gelsolin, are repressed (Hoshikawa et al., 1993; Van Lint C et al., 1996; Saito et al., 1999); whereas, the tumor activators, such as the hypoxia-induced factor-1 (HIF-1) and vascular endothelial growth factor (VEGF), are up-regulated (Kim et al, 2001). One of major causes of these abnormal gene expressions is attributable to the aberrant recruitment of HDAC. Therefore, the inhibition of HDAC activity, as well as the disruption of the HDAC complex, has been recognized as a potent strategy for cancer therapy. In fact, the inhibition of HDAC by HDAC specific inhibitors shows several changes, both at the molecular and cellular level. These include the acetylation state of histone, expression of some genes, as well as the morphology and proliferation of the cell. Hyperacetylated histones that are induced by the HDAC inhibitor change the chromatin structure and lead to the derepression of specific genes that were repressed by HDAC. The expression of these proteins induce growth arrest, detransformation of transformed cells to normal ones, and apoptotic cell death in a variety of transformed cells, as well as neuroblastoma, melanoma, and leukemia cells (Tsuji et al., 1976; Yoshida et al., 1990; Kijima et al., 1993; Newmark et al., 1994; Yoshida et al., 1995; Kwon et al., 1998; Glick et al., 1999; Qui et al., 1999; Lim et al., 2002). In addition, HDACs are overexpressed under specific environmental conditions, such as hypoxia, hypoglycemia, and serum deprivation (Kim et al., 2001) (Fig. 3, C). Among these conditions, hypoxia is one of the key factors to trigger angiogenesis via the induction of angiogenic factors. Angiogenesis, a new blood vessel formation by endothelial cells, is controlled by oppositelyregulating factors that induce or suppress endothelial cell growth. The corruption of this balance is directly related to the outgrowth and spreading of cancer cells (Folkman et al., 1996). We, as well as other groups, have shown that the gene expression regulation through the acetylation of histone is highly involved in the control of angiogenesis (Kim et al., 2001; Kwon et al., 2001; Deroanne et al., 2002). We demonstrated that specific HDAC inhibitors, such as trichostatin A (TSA) and FK228, induce the expression of p53 and von Hippel-Lindau (VHL) under hypoxia conditions; whereas, they reduced the expression of HIF- $1 \alpha$ and VEGF, which resulted in the inhibition of angiogenesis (Kim et al., 2001; Kwon et al., 2002) (Fig. 3, D). Correspondingly, the over-expression of HDAC1 induced angiogenesis, both in vitro and in vivo, through the increased expression of VEGF, as well as the suppression of p53 and VHL. These results demonstrated that HDAC plays a significant role in the hypoxia-induced angiogenesis that is highly related to carcinogenesis.

\section{Non-histone Proteins as New Substrates for HDAC}

Following the characterization of the HDAC function in several biological systems (including carcinogenesis and angiogensis), other substrates of HDAC, besides histone, have gathered interest. Until recently, a number of non-histone proteins were revealed to have acetylation modification. These are transcription factors, such as p53, E2F, transcription factor II E beta (TFIIE $\beta$ ), T-cell transcription factor (TCF), GATA, and nuclear receptor co-activator ACTR (activin receptor) $(\mathrm{Gu}$ et al., 1997; Imfor et al., 1997; Munshi et al., 1998; Zhang et al., 1998; Chen et al., 1999; Marzio et al., 2000) and cytosolic proteins, such as tubulin and importin (Sterner et al., 1979; Bannister et al., 2000; Grozinger et al., 2000; Hubbert et al., 2002). The biological consequence of reversible acetylation in these proteins appears to be determined by the location where the acetylation of the protein occurs. For instance, the acetylation modification of DNA-binding transcription factors, such as p53, E2F1, and GATA, results in increased DNA binding for these proteins. Interestingly, however, the binding of TCF with its co-activator, armadillo, is disrupted by the acetylation of TCF. It is also noteworthy that acetylated E2F1 and tubulin show a longer half-life than that of the nonacetylated form (Takemura et al., 1992; Martinez-Balbas et al., 2000). In addition, the acetylation of importin is involved in the shuttling of the protein from the cytoplasm to the nucleus. All of these results demonstrate that reversible acetylation/deacetylation may occur in a variety of proteins in the cell. Also, HDAC is involved in multiple functions for cellular phenotype, including the binding of protein with DNA, protein-protein interaction, protein stability, and translocation of the protein.

\section{HDAC Structure and Binding Mode of HDAC Inhibitor}

Recently, the Pavletich group determined the X-ray crystal structure of the HDAC catalytic domain (Finnin et al., 1999). An approximately 390-amino acid region that forms the core of the catalytic domain in the hyperthermophilic bacterium Aquifex aeolicus was used for crystallization, since the domain is commonly shared from bacteria to humans. The 
identification of the X-ray crystal structure of HDAC catalytic domain provided the molecular mode of action of HDAC and rationale for the development of more potent HDAC specific inhibitors. The result showed that the catalytic domain of HDAC consisted of a tubular pocket, a zinc-binding site, and two asparagines-histidine charge-relay systems. It was speculated from this enzyme-inhibitor binding structure that TSA-like inhibitors block the HDAC activity through the chelation of zinc ion using the polar group, such as hydroxamic acid or benzamide group at the end of the hydrophobic spacer.

\section{HDAC Inhibitors and Their Role in HDAC Biology and Cancer Therapy}

The HDAC inhibitors play significant roles in the functional study of HDAC. Interestingly, none of these compounds were originally discovered for their ability to inhibit HDAC activity; rather, they were discovered on the basis of their phenotype activity on transformed cells. For example, TSA, a well-known HDAC inhibitor, was originally isolated as an antifungal agent. However, Yoshida et al. discovered the compound as a potent anticancer agent that induces differentiation of murine erythroleukemia cells, and identified HDAC as a molecular target (Yoshida et al., 1995). Likewise, FK228 (formerly depsipeptide or FK901228) and depudecin, were isolated from a screening project for agents that revert the malignant phenotype of H-ras-transformed NIH3T3 cells to normal ones, and identified HDAC as their target (Sugita et al., 1992; Ueda et al., 1994; Kwon et al., 1998). These interesting features in the discovery of HDAC inhibitors have made them one of the best examples for chemical genetics that utilize biologically-active chemicals as probes for functional studies of target genes. Several types of HDAC inhibitors with different chemical structures have been extensively developed from nature and chemical synthesis, based on known HDAC inhibitors and protein structures. These inhibitors are able to divide into 4 classes, based on their functional group for inhibiting HDAC activity and chemical structures (Fig. 4). The first class is the short-chain fatty acids, such as butyrate and phenylbutyrate that are currently used in clinics. But, they are not ideal clinical drugs because of the high concentration (milli-molar) requirement for the inhibition of HDAC (Newmark et al., 1994; Carducci et al., 1997). Next are the aliphatic compounds that have hydroxamic acid, such as TSA, suberolyanilide hydroxamic acid (SAHA), and oxamflatin. This compound class is considered to exhibit their inhibiting activity by chelating the zinc ion at the catalytic site of HDAC, as previously described. In the case of SAHA, the chemical structure is basically similar to TSA, but differs in that it was generated from chemical synthesis. Although SAHA shows weaker inhibitory activity for HDAC than TSA, it is effective for the inhibition of tumor growth, both in vitro and in vivo, at the micromolar concentration (Richon et al., 1998; Glick et al., 1999; Butler et al., 2000). Furthermore, SAHA reduced tumor volume by $78 \%$, and showed no side effects in animal studies. This makes it one of the promising HDAC inhibitors as an antitumor drug (Cohen et al., 1999). The third class is the cyclic tetrapeptide compounds that have epoxy ketone or sulfohydryl group as a functional group. Trapoxin (TPX) and FK228 fall into this class (Kijima et al., 1993; Ueda et al., 1994). The epoxy ketone moiety of TPX forms an irreversible complex with the nucleophilic amino acid residue in the catalytic pocket of HDAC. This property of the compound was used in the affinity-matrix-based purification of HDAC (Taunton et al., 1996). Notably, FK228 is a clinically important HDAC inhibitor. FK228 has a unique structure with cyclic peptide that has a disulfide bridge structure in the molecule. Interestingly, Furumai et al. reported recently that the disulfide bond can be reduced in the cell by the cellular reducing agent, such as glutathione, then the reduced FK228 effectively inhibits the HDAC activity without losing its inhibitory activity before binding with HDAC (Furumai et al., 2002). Therefore, FK 228 is a kind of natural prodrug that provides a new insight for developing HDAC inhibitors as anti-cancer agents. Another interesting compound is CHAP31. It is one of the synthetic cyclic peptide compounds that have a hybrid chemical structure of both TSA and TPX. This compound has tetracyclic peptide, like TPX, but the functional group of TPX, epoxyketone, was replaced with hydroxamic acid from TSA. The final class is the benzamidebearing compounds, such as MS-27-275 and CI-994 (Saito et al., 1999; Prakash et al., 2001). Interestingly, MS-27-275 showed strong antitumor activity with low toxicity in nude mice, and is under the Phase I clinical trial. Among several HDAC specific inhibitors from nature and synthetic sources, phenylbutyrate and valproic acid (short chain fatty acids), CI994 and MS-27-275 (benzamides), SAHA (hydroxamic acid) and FK228 (cyclic peptide), are currently in clinical development. These agents are undergoing the Phase I or Phase II evaluations, both along and in combination with cytotoxic or differentiation inducing agents, which supports the crucial role of HDAC in carcinogenesis. Although several structurally-unrelated HDAC inhibitors have been developed, there have been no studies on the inhibitor that specifically inhibits HDAC isotypes. Given the number and tissue specific expression of HDAC in humans, HDAC inhibitors with isotype specific inhibitory activity will be required for a better understanding of the HDAC function and the development of HDAC inhibitor cancer therapy. Recently, the Schreiber and Jung group reported a generation of chemical libraries that target the isotype specific inhibition of HDAC (Sternson et al., 2001; Wittich et al., 2002). It is also noteworthy that FK228 showed a more specific inhibition on the class I HDAC activity than that of the class II HDAC (Furumai et al., 2002). Since the amino acid resides around the rim of the catalytic pocket in HDAC differ from classes I and II, the isotypespecific-HDAC inhibitors can be developed from the 


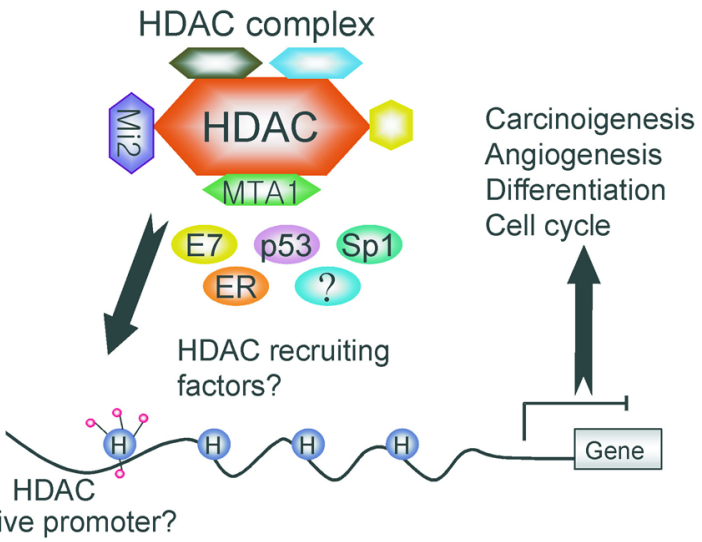

Fig. 6. Model of HDAC function in cells. The HDAC complex is recruited by specific recruiting factors into a specific promoter. The aberrant recruitment of the HDAC complex that represses the transcription of specific genes, such as the tumor suppressor, leads to the occurrence of carcinogenesis and angiogenesis. In addition to the transcriptional regulation, HDAC plays an important role in several interactions (such as protein-DNA interaction, protein-protein interaction, and protein stability) by changing the acetylation status of the proteins.

compounds that have a high affinity with the amino acids at the rim. These isotype-specific-HDAC inhibitors will provide more selective and lower toxic agents for the HDAC inhibitor cancer therapy. In addition, these inhibitors will be useful tools for the chemical genetic study of the HDAC isotype, as shown in the case of other HDAC inhibitors as well as the Sir2 specific inhibitor that was recently developed (Bernstein et al., 2000).

Another possible challenge is to develop the compound that inhibits the formation of the HDAC-complex, but not the HDAC activity (Fig. 5). Recently, Pflum et al. reported that the highly charged-carboxyl-terminal region of HDAC is essential for HDAC activity. The mutation of S421 and S423 to alanine, or the disruption of the caseine kinase 2 (CK2), abolishes the phosphorylation of serine residues in the carboxyl terminus of HDAC, which results in decreased HDAC activity and complex formation with MTA1, a component of the NuRD complex. In addition, Galasinski et al. reported that the disruption of the phosphorylation affects the complex formation in all type of co-repressors. The interaction between HDAC1 and the co-repressors, mSin3A and YY1, was affected by the phosphorylation of HDAC1, but not between HDAC and RbAp46/48. These results suggest the significant role of the HDAC complex formation in the HDAC function. The discovery of an inhibitor that disrupts the interaction between HDAC and the specific interacting protein will be another challenge for targeting the HDAC function. It will also provide new tools to elucidate the role of the HDAC complex in the cell.

\section{Concluding Remarks}

Despite the early perception of the significance of the reversible acetylation modification on histone, most of the major progress in this event began with the isolation of the specific HDAC inhibitors and the cloning of the enzymes. To date, eleven HDAC isotypes have been identified from humans, and the biological role of HDAC in several cellular functions (including cancer) has been revealed, as described previously. As result, HDAC is recognized as one of the promising targets for the development of anticancer drugs. However, there are still many uncovered mysteries of the HDAC function, such as the regulation mechanism of the specific gene expression and the recruiting factors for the HDAC complex to the specific chromosome region to confer HDAC activity. In addition, the HDAC role in several interactions (such as protein-DNA interaction, protein-protein interaction, and protein stability) will be another significant issue to be elucidated (Fig. 6). Novel HDAC inhibitors that have properties of the isotype specific inhibition or blockingHDAC complex will be powerful tools for this purpose, the same as conventional HDAC inhibitors have done for the study of HDAC. In conclusion, HDAC is a crucial factor in carcinogenesis, as well as a promising target for the development of anticancer drugs and therapies.

Acknowledgments This work was supported by grant No. R01-1999-00121-0 from the Korea Science \& Engineering Foundation to HJK. We thank our group members who were involved in the HDAC and related project for their dedicated contributions. They are Joong Sup Shim, Hye Jin Jung, Ji Yong Lee, $\mathrm{Na}$ Yeon $\mathrm{Ku}$, Si Na Kim, Seung Young Kim, Sung Eun Hwang, Jin Hee Kim, Young Mi Song, Hye Jin Kim, and Hye Won Kim.

\section{References}

Ahringer, J. (2000) NuRD and SIN3: histone deacetylase complexes in development. Trends Genet. 16, 351-356.

Alland, L., Muhie, R., Hou Jr., H., Potes, J., Chin, L., SchreiberAgus, N., and DePinho, R. A. (1997) Role for N-CoR and histone deacetylase in Sin3-mediated transcriptional repression. Nature 387, 49-55.

Bannister, A. J., and Kouzarides, T. (1996) The CBP co-activator is a histone acetyltransferase. Nature 384, 641-643.

Bannister, A. J., Miska, E., Gorlich, D., and Kouzarides, T. (2000) Acetylation of importin-alpha nuclear import factors by CBP/ p300. Curr. Biol. 10, 467-70.

Bernstein, B. E., Tong, J. K., and Schreiber, S. L. (2000) Genomewide studies of histone deacetylase function in yeast. Proc. Natl. Acad. Sci. USA 97, 13708-13713.

Butler, L. M., Higgins, B., Fox, W. D., Agus, D. B., CordonCardo, C., and Scher, H. J. (2000) Hybrid polar inhibitors of 
histone deacetylase suppress the growth of the CWR22 human prostate cancer xenograft. Proc. Am. Assoc. Cancer Res. 41, Abstract 2396.

Carducci, M., Bowling, M. K., Eisenberger, M., Sinibaldi, V., Chen, T., and Nor, D. (1997) Phenylbutyrate (PB) for refractory solid tumors: Phase I clinical and pharmacologic evaluation of intravenous and oral PB. Anticancer Res. 17, 3972-3973.

Chen, H., Lin, J. R., Xie, W., Wilpitz, D., and Evans, M. R. (1999) Regulation of hormone-induced histone hyperacetylation and gene activation via acetylation of an acetylase. Cell 98, 675-686.

Choi, J. H., Kwon, H. J., Yoon, B. I., Kim, J. H., Han, S. U., Joo, H. J., and Kim, D. Y. (2001) Expression Profile of Histone Deacetylase1 in Gastric Cancer Tissues. Jpn. J. Cancer Res. 92, 1300-1304.

Cohen, L. A., Amin, S., Marks, P. A., Rifkind, R. A., Desai, D., and Richon, V. M. (1999) Chemoprevention of carcinogeninduced mammary tumorigenesis by the hybrid polar cytodifferentiation agent, suberanilohydroxamic acid (SAHA). Anticancer Res. 19, 4999-5005.

Cress, W. D., and Seto, E., (2000) Histone deacetylases, transcriptional control, and cancer. J. Cell. Physiol. 184, 1-16.

Davie, J. R. (1998) Covalent modifications of histones: expression from chromatin templates. Curr. Opin. Genet. Dev. 8, 173-178.

Deroanne, C. F., Bonjean, K., Servotte, S., Devy, L., Colige, A., Clausse, N., Blacher, S., Verdin, E., Foidart, J. M., Nusgens, B. V., and Castronovo, V. (2002) Histone deacetylases inhibitors as anti-angiogenic agents altering vascular endothelial growth factor signaling. Oncogene 21, 427-436.

Dhordain, P., Lin, R. J., Quief, S., Lantoine, D., Kerckaert, J. P., Evans, R. M., and Albagli, O. (1998) The LAZ3 (BCL06) oncoprotein recruits a SMRT/Msin3A/histone deacetylase containing complex to mediate transcriptional repression. Nucleic Acids Res. 26, 4645-4651.

Finnin, M. S., Donigian, J. R., Cohen, A., Richon, V. M., Rifkind, R. A., Marks, P. A., and Pavletich, N. P. (1999) Structures of a histone deacetylase homologue bound to the TSA and SAHA inhibitors. Nature 401, 188-193.

Folkman, J. (1996) Fighting cancer by attacking its blood supply. Sci. Am. 275, 150-154.

Furumai, R., Matsuyama, A., Kobashi, N., Lee, K. H., Nishiyama, M., Nakajima, H., Tanaka, A., Komatsu, Y., Nishino, N., Yoshida, M., and Horinouchi, S. (2002) FK228(depsipeptide) as a natural prodrug that inhibits class I histone deacetylase. Cancer Res. 62, 4916-4921.

Galasinski, S. C., Resing, K. A., Goodrich, J. A., and Ahn, N. G. (2002) Phosphatase inhibition leads to histone deacetylase 1 and 2 phosphorylation and disruption of co-repressor interaction. J. Biol. Chem. 277, 19618-19626.

Glick, R. D., Swendeman, S. L., Coffey, D. C., Ridkind, R. A., Marks, P. A., and Richon, V. M. (1999) Hybrid polar histone deacetylase inhibitor induces apoptosis and CD95/CD95 ligand expression in human neuroblastoma. Cancer Res. 59, 43924399.

Grozinger, C. M., Hassig, C. A., and Schreiber, S. L. (1999) Three proteins define a class of human histone deacetylases related to yeast Hda1p. Proc. Natl. Acad. Sci. USA 96, 48684873.

Grozinger, C. M., and Schreiber, S. L. (2000) Regulation of histone deacetylases 4 and 5 and transcriptional activity by 143-3-dependent cellular localization. Proc. Natl. Acad. Sci. USA 97, 7835-7840.

Gu, W., and Roeder, R. G. (1997) Activation of p53 sequencespecific DNA binding by acetylation of the p53 C-terminal domain. Cell 90, 595-606.

Guschin, D., Wade, P. A., Kikyo, N., and Wolffe, A. P. (2000) ATP-dependent histone octamer mobilization and histone deacetylation mediated by the Mi-2 chromatin remodeling complex. Biochemistry 39, 5238-5245.

Hassig, C. A., Fleischer, T. C., Billin, A. N., Schreiber, S. L., and Ayer, D. E. (1997) Histone deacetylase activity is required for full transcriptional repression by mSin3A. Cell 89, 341-347.

Heizel, T., Lavinsky, R. M., Mullen, T., Soderstrome, M., Laherty, C. D., Torchia, J., Yang, W., Brard, G., Ngo, S. D., Davie, J. R., Seto, E., Eisenman, R. N., Rose, D. W., Glass, C. K., and Rosenfeld, M. G. (1997) A complex containing N-CoR, mSin3 and histone deacetylase mediates transcriptional repression. Nature 387, 43-48.

Hoshikawa, Y., Kwon, H. J., Yoshida, M., Horinouchi, S., and Beppu, T. (1994) Trichostatin A induces morphological changes and gelsolin expression by inhibiting histone deacetylase in human carcinoma cell lines. Exp. Cell Res. 214, 189-197.

Huang, E. Y., Zhang, J., Miska, E. A., Guenther, M. G., Kouzarides, T., and Lazar, M. A. (2000) Nuclear receptor corepressor partner with class II histone deacetylases in a Sin3independent repression pathway. Genes Dev. 14, 45-54.

Hubbert, C., Guardiola, A., Shao, R., Kawaguchi, Y., Ito, A., Nixon, A., Yoshida, M., Wang, X. F., Yao, T. P. (2002) HDAC6 is a microtubule-associated deacetylase. Nature 417, 455-458.

Hwang, H. S., Suh, N. Y. and Song, K. W. (2000) The fission yeast $\mathrm{Hda} 1 \mathrm{p}$ functions on the regulation of proper cell division. J. Biochem. Mol. Biol. 33, 263-267.

Imfor, A., Yang, X. J., Ogryzko, V. V., Nakatani, Y., Wolffe, A. P., and Ge, H. (1997) Acetylation of general transcription factors by histone acetyltransferase. Curr. Biol. 7, 689-692.

Kadosh, D., and Struhl, K. (1997) Repression by Ume6 involves recruitment of a complex containing $\operatorname{Sin} 3$ co-repressor and Rpd3 histone deacetylase to target promoters. Cell 89, 365-371.

Khochbin, S., and Kao, H. (2001) Histone deacetylase complexes: functional entities or molecular reservoirs. FEBS Lett. 494, 141-144.

Kijima, M., Yoshida, M., Sugita, K., Horinouchi, S., and Beppu, T. (1993) Trapoxin, an antitumor cyclic tetrapeptide, is an irreversible inhibitor of mammalian histone deacetylase. J. Biol. Chem. 268, 22429-22435.

Kim, M. S., Kwon, H. J., Lee, Y. M., Baec, J. H., Jang, J. E., Lee, S. W., Moon, E. J., Kim, H. S., Lee, S. K., Chung, H. Y., Kim, C. W., and Kim, G. W. (2001) Histone deacetylases induce angiogenesis by negative regulation of tumor suppressor genes. Nature Med. 7, 437-443.

Kornberg, R. D. (1974) Chromatin structure: a repeating unit of histones and DNA. Science 184, 868-871.

Kornberg, R. D., and Lorch, Y., (1999) Twenty-five years of the Nucleosome, fundamental particle of the eukaryote chromosome. Cell 98, 285-294.

Kouzarides, T. (1999) Histone acetylase and deacetylase in cell proliferation. Curr. Opin. Genet. Dev. 9, 40-48. 
Kwon, H. J., Kim, M. S., Kim, M. J., Nakajiam, H., and Kim, K. W. (2002) Histone deacetylase inhibitor FK228 inhibits tumor angiogenesis. Int. J. Cancer 97, 290-296.

Kwon, H. J., Kim, M. J., and Kim, S. Y. (2002) New Yeast CellBased Assay System for Screening of Histone Deacetylase1 Complex Disruptor. J. Microbiol. Biotech. 12, 286-291.

Kwon, H. J., Owa, T., Hassig, C. A., Shimada, J., and Schreiber, S. L. (1998) Depudecin induces morphological reversion of transformed fibroblasts via the inhibition of histone deacetylase. Proc. Natl. Acad. Sci. USA 95, 3356-3361.

Laherty, C. D., Yang, W., Sun, J., Davie, J. R., Seto, E., and Eisenman, R. N. (1997) Histone deacetylases associated with the $\mathrm{mSin} 3$ co-repressor mediate Mad transcriptional repression. Cell 89, 349-356.

Lander, J., Sutton, A., Tafrov, S. T., Heller, R. C., Stebbin, J., Pillus, L., and Sternglanz, R. (2000) The silencing protein SIR2 and its homologs are NAD-dependent protein deacetylases. Proc. Natl. Acad. Sci. USA 97, 5807-5811.

Lemercier, C., Verdel, A., Galloo, B., Curtet, S., Brocard, M., and Khochbin, S. (2000) mHDA1/HDAC5 histone deacetylase interacts with and represses MEF2A transcriptional activity. $J$. Biol. Chem. 275, 15594-15599.

Lim, Y., Han, I., Kwon, H. J., and Oh, E. S. (2002) Trichostatin A-induced detransformation correlates with decreased focal adhesion kinase phosphorylation at tyrosine 861 in rastransformed fibroblasts. J. Biol. Chem. 277, 12735-12740.

Luger, K., Mader, A. W., Richmond, R. K., Sargent, D. F., and Richmond, T. J. (1997) Crystal structure of the Nucleosome core particle at 2.8A resolution. Nature 389, 251-260.

Luo, R. W., Postigo, A. A., and Dean, D. C. (1998) Rb interacts with histone deacetylase to repress transcription. Cell 92, 463473.

Mahoney, M. G., Simpson, A., Jost, M., Noe, M., Kari, C., Pepe, D., Choi, Y. W., Uitto, J., and Rodeck, U. (2002) Metastasisassociated protein 1 (MTA1) enhances migration, invasion, and anchorage-independent survival of immortalized human keratinocytes. Oncogene 21, 2161-2170.

Marks, P. A., and Rifkind, R. A. (1978) Erythroleukemic differentiation. Annu. Rev. Biochem. 47, 419-448.

Marks, P. A., Rifkind, R. A., Richon, V. M., Breslow, R., Miller, T., and Kelly, W. K. (2001) Histone deacetylase and cancer: causes and therapies. Nature Rev. Cancer 1, 194-202.

Martinez-Balbas, M., Bauere, U. M., Nielsen, S. J., Brehm, A., and Kouzarides, T. (2000) Regulation of E2F activity by acetylation. EMBO J. 19, 662-671.

Marzio, G., Wagenre, C., Gutierrez, M. I., Cartwright, P., Helin, K., and Giacca, M. (2000) E2F family members are differentially regulated by reverse acetylation. J. Biol. Chem. 275, 10887-10892.

Mazumdar, A., Wang, R., Mishra, S. K., Adam, L., BagheriYarmand, R., Mandal, M., Vadlamudi, R. K., and Kumar, R., (2001) Transcriptional repression of oestrogen receptor by metastasis-associated protein 1 co-repressor. Nature Cell Biol. 3, 30-37.

Mizzen, C. A., Yang, X. J., Kokubo, T., Brownell, J. E., Bannister, A. J., and Owen-Hughes, T. (1996) The TAF (II) 250 subunit of TF(II)D has histone acetyltransferase activity. Cell 87, 12611270.

Munshi, N., Merika, M., Yie, J., Senger, K., Chen, G., and Thanos, D. (1998) Acetylation of HMG1 (Y) by CBP turns off
IFNB expression by disrupting the enhanceosome. Mol. Cell 2, 457-467.

Nagy, L., Kao, H., Chakravarti, D., Lin, R., Hassg, C. A., Ayer, D. E., Schreiber, S. L., and Evans, R. M. (1997) Nuclear receptor repression mediated by a complex containing SMRT, mSin3A and histone deacetylase. Cell 89, 373-380.

Nan, X., Ng, H., Johnson, C. A., Laherty, C. D., Turner, B. M., Eisenman, R. N. and Bird, A. (1998) Transcriptional repression by the methl-CpG-binding protein $\mathrm{MeCP} 2$ involves a histone deacetylase complex. Nature 393, 386-389.

Newmark, H. L., Lupton, J. R. and Young, C. W. (1994) Butyrate as a differentiating agent; pharmacokinetics, analogues and current status. Cancer Lett. 78, 1-5.

Ogryzko, V. V., Schiltz, R. L., Russanova, V., Howard, B. H. and Nakatino, Y. (1996) The transcriptional co-activators p300 and CBP are histone acetyltransferase. Cell 87, 953-959.

Pandolfi, P. P. (2001) Transcription therapy for cancer. Oncogene 20, 3116-3127.

Pflum, M. K., Tong, J. K., Lane, W. S., and Schreiber, S. L. (2001) Histone deacetylase 1 phosphorylation promotes enzymatic activity and complex formation. J. Biol. Chem. 276, 47733-47741.

Petrij, F., Giles, R. H., Dauwerse, H. G., Saris, J. J., Hennekam, R. C., Masuno, M., Tommerup, N., van Ommen, G. J., Goodman, R. H., and Peters, D. J. (1995) Rubinstein-Taybi syndrome caused by mutations in the transcriptional coactivator CBP. Nature 376, 348-351.

Prakash, S., Foster, B. J., Meyer, M., Wozniak, A., Heibrum, L. K., Flaherty, L., Zalupski, M., Radulovic, L., Valdivieso, M., and LoRusso, P. M. (2001) Chronic oral administration of CI994: a Phase I study. Investg. New Drugs 19, 1-11.

Qui, L., Kelso, M. J., Hansen, C., West, M. L., Fairlie, D. P., and Parsons, P. G. (1999) Anti-tumor activity in vitro and in vivo of selective differentiating agnets containing hydroxamate. $\mathrm{Br} . \mathrm{J}$. Cancer 80, 1252-1258.

Richon, V. M., Emiliani, S., Verdin, E., Webb, Y., Breslow, R., and Rifkind, R. A. (1998) A class of hybrid polar inducers of transformed cell differentiation inhibits histone deacetylase. Proc. Natl. Acad. Sci. USA 95, 3003-3007.

Saito, A., Yamashita, T., Mariko, Y., Nosaka, Y., Tsuchiya, K., and Ando, T. (1999) A synthetic inhibitor of histone deacetylase, MS-27-275, with marked in vivo antitumor activity against human tumors. Proc. Natl. Acad. Sci. USA 96, 4592-4597.

Spencer, T. E., Jenster, G., Burcin, M. M., Allis, C. D., Zhou, J., and Mizzen, C. A. (1997) Steroid receptor co-activator-1 is a histone acetyltransferase. Nature 389, 194-198.

Sterner, R., Vidali, G., and Allfrey, V. G. (1979) Studies of acetylation and deacetylation in high mobility group proteins. Identification of the sites of acetylation in HMG-1. J. Biol. Chem. 254, 11577-11583.

Sternson, S. M., Wong, J. C., Grozinger, C. M., and Schreiber, S. L. (2001) Synthesis of 7200 small molecules based on a substructural analysis of the histone deacetylase inhibitors trichostatin and trapoxin. Org. Lett. 3, 4239-4242.

Struhl, K. (1998) Histone acetylation and transcriptional regulatory mechanisms. Genes Dev. 12, 599-606.

Sugita, K., Yoshida, H., Matsumoto, M., and Matsutani, S. (1992) A novel compound, depudecin, induces production of transformation to the flat phenotype of NIH3T3 cells transformed by ras-oncogene. Biochem. Biophys. Res. Commun. 
182, 379-387.

Takemura, R., Okabe, S., Umeyama, T., Kanai, Y., Cowan, N. J., and Hirokawa, N. (1992) Increased microtubule stability and átubulin acetylation in cells transfected with microtubuleassociated proteins MAP1B, MAP2 or tau. J. Cell Sci. 103, 953-964.

Taunton, J., Hassig, C. A., and Schreiber, S. L. (1996) A mammalian histone deacetylase related to the yeast transcriptional regulator Rpd3p. Science 272, 408-411.

Toh, Y., Pencil, S. D., and Nicolson, G. L., (1998) A novel candidate metastasis-associated gene, mta1, differentially expressed in highly metastatic mammary adenocarcinoma cell lines. J. Biol. Chem. 269, 22958-22963.

Tong, J. K., Hassig, C. A., Schnitzler, G. R., Kingston, R. E., and Schreiber, S. L. (1998) Chromatin deacetylation by an ATPdependent nucleosome remodeling complex. Nature 395, 917921.

Tsuji, N., Kobaysshi, M., Nagashima, K., Wakisaka, Y., and Koizumi, K. (1976) A new antifungal antibiotics, trichostatin. $J$. Antibiot. (Tokyo). 29, 1-6.

Ueda, H., Nakajima, H., Hori, Y., Goto, T., and Okuhara, M. (1994) Action of FR901228, a novel antitumor bicyclic depsipeptide produced by Chromobacterium violaceum no. 968, on Ha-ras transformed NIH3T3 cells. Biosci. Biotechnol. Biochem. 58, 1579-1583.

Van Lint, C., Emiliani, S., and Verdin, E. (1996) The expression of a small fraction of cellular gene is changed in response to histone hyperacetylation. Gene Expr. 5, 245-254.

Wittich, S., Scherf, H., Xie, C., Brosch, G., Loidl, P., Gerhauser, C., and Jung, M. (2002) Structure-activity relationships on phenylalanine-containing inhibitors of histone deacetylase: in vitro enzyme inhibition, induction of differentiation, and inhibition of proliferation in Friend leukemic cells. J. Med. Chem. 45, 3296-3309.

Yang, W., Inouye, C., Zeng, Y., Bearss, D., and Seto, E. (1996) Transcriptional repression by YY1 is mediated by interaction with a mammalian homolog of the yeast global regulator RPD3. Proc. Natl. Acad. Sci. USA 93, 12845-12850.

Yoshida, M., Horinouchi, S., and Beppu, T. (1995) Trichostatin A and trapoxin: novel chemical probes for the role of histone acetylation in chromatin structure and function. Bioessays 17, 423-430.

Yoshida, M., Kijima, M., Akita, M., and Beppu, T. (1990) Potent and specific inhibition of mammalian histone deacetylase both in vivo and in vitro by trichostatin A. J. Biol. Chem. 265, 17174-17179.

Zhang, W., and Bieker, J. J. (1998) Acetylation and modulation of erythroid Kruppel-like factor (EKLF) activity by interaction with histone acetyltransferase. Proc. Natl. Acad. Sci. USA 95, 9855-9860.

Zhang, Y., Ng, H., Erdjument-Bromage, H., Tempst, P., Bird, A., and Reinberg, D. (1999) Analysis of the NuRD subunits reveals a histone deacetylases core complex and a connection with DNA methylation. Genes Dev. 13, 1924-1935. 